Pacific Journal of Mathematics

FLOWS AND NONCOMMUTING PROJECTIONS ON HILBERT

FRANK HERBERT BRowNELL, I 


\section{FLOWS AND NONCOMMUTING PROJECTIONS ON HILBERT SPACE}

F. H. BROWNELL

1. Introduction. Let $\{E(A)\}$ over $A \in B_{1}$, the Borel subsets of the real line $R_{1}$, be a resolution of the identity for the Hilbert space $X$, and consider the flow

$$
u_{t}=U_{t} u_{0}=\int_{-\infty}^{L} e^{i t \lambda} d E(\lambda) u_{0},
$$

over $t$ real for fixed $u_{0} \in X$. Let $P$ be an orthogonal projection in $X$. Our problem is to study the asymptotic behavior of $\left\|P u_{t}\right\|^{2}$ as $t \rightarrow+\infty$ or $t \rightarrow-\infty$. If $P$ commutes with all $E(A)$, then

$$
P U_{t}=U_{t} P \text { and }\left\|P u_{t}\right\|^{2}=\left\|P u_{0}\right\|^{2} \text {, }
$$

a constant, so we are interested only in the case where $P$ fails to commute.

It is easy to see that this asymptotic behavior depends upon the nature of $\gamma$ through the equation

$$
\left\|P u_{t}\right\|^{2}=\int_{R_{2}} e^{i t(x-y)} d y(x, y)
$$

integrated in a Riemann sense over the plane $R_{2}$, where

$$
\gamma(A \times B)=\left(P E(A) u_{0}, E(B) u_{0}\right) .
$$

If $\gamma$ admits a $\sigma$-additive and bounded extension over $B_{2}$, the Borel sets of $R_{2}$, standard procedures enable us to say that $\left\|P u_{t}\right\|^{2}$ converges densely to $C$ as $t \rightarrow+\infty$ or $t \rightarrow-\infty$ if and only if $\gamma\left(D_{s}\right)=0$ for $s \neq 0$ and $\gamma\left(D_{0}\right)=C$, where the diagonal

$$
D_{s}=\left\{\left(x_{s} y\right) \in R_{2} \mid x-y=s\right\} .
$$

The interesting fact here is, as we shall see by example, that $\gamma$ need not in general be either $\sigma$-additive or bounded, although it is always both if $P$ is

Received July 23, 1953.

Pacific J. Math. 5 (1955), 1-16 
compact. Also if $\gamma$ is not both $\sigma$-additive and bounded, it can happen that $\left\|P u_{t}\right\|^{2} \rightarrow C$ densely as $t \rightarrow+\infty$ and $\rightarrow C^{\prime}$ densely as $t \rightarrow-\infty$ with $C \neq C^{\prime}$.

7 hiese results, particularly the last, seem to be of some interest in quantum physics. Here the vector $u_{t}=U_{t} u_{0}$ represents the state of the physical system at time $t$ for the initial state $u_{0}$; and, for $\left\|u_{0}\right\|^{2}=1$, we can take $\left\|P u_{t}\right\|^{2}$ to the probability of the system at time $t$ being in the situation which corresponds to the range space of $P$.

2. Principal results. As above, let $E(A)$ be a spectral measure [4, p. 58] over $A \in B_{1}$ for the Hilbert space $X$. Thus

$$
\int_{R_{1}}^{L} e^{i t \lambda} d E(\lambda) u_{0}
$$

exists as the $X$ norm limit of the Lebesgue sums for every $u_{0} \in X$, and we define

$$
u_{t}=U_{t} u_{0}=\int_{R_{1}}^{L} e^{i t \lambda} d E(\lambda) u_{0}
$$

so $U_{t}$ is a unitary operator on $X$. Also since each term in a Lebesgue sum for $e^{i t \lambda}$ corresponds to a countable disjoint union of equal intervals equally spaced

$$
\int_{R_{1}}^{L} e^{i t \lambda} d E(\lambda) u_{0}=\int_{R_{1}}^{R} e^{i t \lambda} d E(\lambda) u_{0}
$$

for the latter defined as the $X$ norm limit of Riemann sums. Thus for $P$ an orthogonal projection,

$$
\left\|P u_{t}\right\|^{2}=\left(P u_{t}, u_{t}\right)=\left(\int_{R_{1}}^{R} e^{i t x} d P E(x) u_{0}, \int_{R_{1}}^{R} e^{i t y} d E(y) u_{0}\right)
$$

although $P E(A)$ is generally not a projection. Define for a given $u_{0} \in X$ the complex-valued set function $\gamma$ by

$$
\gamma(A \times B)=\left(P E(A) u_{0}, E(B) u_{0}\right),
$$

so that $y$ clearly has a unique, finitely additive extension over the finite algebra generated by product Borel sets, which includes the finite algebra $\mathcal{F}$ of finite unions of intervals of $R_{2},([3, p .149,(8)]$. Using the continuity of the inner product we thus see that 


$$
\left\|P u_{t}\right\|^{2}=\int_{R_{2}} e^{i t(x-y)} d y(x, y)
$$

where the integral on the right is of the Riemann type defined as the limit as $h, l \longrightarrow 0^{+}, h N, l M \longrightarrow+\infty$ of the Riemann sums

$$
\sum_{n=-N}^{N} \sum_{m=-M}^{M} e^{i t\left(x_{n}-y_{m}\right)} \gamma\left(A_{n} \times B_{m}\right),
$$

with

$$
A_{n}=((n-1) h, n h] \text { and } B_{m}=((m-1) l, m l]
$$

and $x_{n} \in A_{n}$ and $y_{m} \in B_{m}$ arbitrarily chosen. We shall call a complex-valued set function over $\mathcal{F}$ tractable if it is both $\sigma$-additive and bounded over $\mathcal{F}$, and thus also of bounded variation over $\mathcal{F}$. For tractable $\gamma$ we have the Jordan decomposition of $\gamma$ into its four nonnegative variations, each one of which is clearly $\sigma$-additive over $\mathcal{F}$. Thus, applying the well-known Borel-Hopf extension theorem [3,p.49-54], we see that $\gamma$ possesses a unique, $\sigma$-additive, and bounded extension at least over $B_{2}$, the Borel subsets of $R_{2}=R_{1} \times R_{1}$. Thus applying dominated convergence to the Riemann sums, for tractable $\gamma$ we have

$$
\int_{R_{2}}^{R} e^{i t(x-y)} d y(x, y)=\int_{R_{2}}^{L} e^{i t(x-y)} d y(x, y)
$$

defined in the ordinary Lebesgue sense. Hence letting

$$
D_{s}=\left\{(x, y) \in R_{2} \mid x-y=s\right\},
$$

the diagonal in $R_{2}$ having $x$ intercept $s$, and

$$
\tilde{\gamma}(A)=\gamma\left(\underset{s \in A}{\bigcup} D_{s}\right)
$$

we see that $\tilde{\gamma}$ is bounded and $\sigma$-additive over $B_{1}$ and (2.1) becomes

$$
\left\|P u_{t}\right\|^{2}=\int_{R_{1}}^{L} e^{i t s} d \tilde{\gamma}(s)
$$

for tractable $\gamma$.

LemMA 1. If $P$ is compact (that is, the range space of $P$ has some finite 
dimension $k)$, then $\gamma$ is tractable, and in fact $(\operatorname{var} \gamma) \leq k\left\|u_{0}\right\|^{2}$.

Proof. Clearly the orthogonal projection $P$ is compact if and only if the unit sphere of the range space is compact, or thus equivalently the range space has some finite dimension $k$. Hence taking $\left\{v_{p}\right\}$ a complete orthonormal system in the range space of $P$, we see that

$$
P u=\sum_{p=1}^{k}\left(u, v_{p}\right) v_{p}
$$

so

$$
\gamma(A \times B)=\sum_{p=1}^{k}\left(E(A) u_{0}, v_{p}\right) \overline{\left(E(B) u_{0}, v_{p}\right)}
$$

for $A, B \in B_{1}$. But $E(A)$ being a spectral measure over $A \in B_{1}$,

$$
\eta_{p}(A)=\left(E(A) u_{0}, v_{p}\right)
$$

has $\eta_{p}$ a $\sigma$-additive, complex-valued set function over $B_{1}$ which is bounded,

$$
\left(\operatorname{var} \eta_{p}\right) \leq\left\|u_{0}\right\|\left\|v_{p}\right\|=\left\|u_{0}\right\| .
$$

Thus

$$
\gamma=\sum_{p=1}^{k} \eta_{p} \times \bar{\eta}_{p}
$$

is a finite sum of product measures of this sort, and hence is $\sigma$-additive and bounded over $B_{2} \supseteq \mathcal{F}$,

$$
(\operatorname{var} \gamma) \leq \sum_{p=1}^{k}\left(\operatorname{var} \eta_{p}\right)^{2} \leq k\left\|u_{0}\right\|^{2} ;
$$

this completes the proof.

As usual we say that a complex-valued, measurable function $f(t)$ converges densely to $C$ as $t \rightarrow+\infty$ if, for every $\rho>0$,

$$
0=\lim _{T \rightarrow \infty} \frac{1}{T} \mu_{1}([0, T] \cap\{t|| f(t)-C \mid \geq \rho\}),
$$


where $\mu_{1}$ is ordinary Lebesgue measure on $R_{1}$, and similarly as $t \rightarrow-\infty$ if

$$
0=\lim _{T \rightarrow \infty} \frac{1}{T} \mu_{1}([-T, 0] n\{t|| f(t)-C \mid \geq \rho\}) .
$$

We collect some well-known results $[2$, p. 25, Theorem 8$]$ in the following lemmas.

Lemma 2. A bounded, measurable $f(t)$ converges densely to $C$ as $t \rightarrow+\infty$ if and only if

$$
0=\lim _{T \rightarrow \infty}\left(\frac{1}{T} \int_{0}^{T}|f(t)-C|^{2} d t\right)
$$

and similarly as $t \longrightarrow-\infty$ if and only if

$$
0=\lim _{T \rightarrow \infty} \frac{1}{T} \int_{-T}^{0}|f(t)-C|^{2} d t .
$$

LEMma 3. If

$$
f(t)=\int_{R_{1}}^{L} e^{i t s} d \phi(s)
$$

where $\phi$ is a bounded and $\sigma$-additive complex-valued set function over $B_{1}$, then $f(t)$ converges densely to $C$ as $t \rightarrow+\infty$ if and only if $\phi(\{s\})=0$ for $s \neq 0$ and $\phi(\{0\})=C$, and the same is true as $t \rightarrow-\infty$.

Proofs. For Lemma 2, if we let

$$
A_{T, \rho}=[0, T] \cap\{t|| f(t)-C \mid \geq \rho\},
$$

and

$$
|f(t)| \leq M<+\infty
$$

for the bound of $f(t)$, we clearly get the result from the following inequalities:

$$
\rho^{2}\left(\frac{1}{T} \mu_{1}\left(A_{T, \rho}\right)\right) \leq \frac{1}{T} \int_{0}^{T}|f(t)-C|^{2} d t \leq \rho^{2}+(M+|C|)^{2}\left(\frac{1}{T} \mu_{1}\left(A_{T, \rho}\right)\right) .
$$

For Lemma 3, we note that the Fubini theorem applies. Thus if we define 


$$
f_{1}(t)=f(t)-C=\int_{R_{1}}^{L} e^{i t s} d \phi_{1}(s) \text { for } \phi_{1}(A)=\phi(A)-C \delta_{0}(A),
$$

and,

$$
\left.\delta_{0}(A)=1 \text { if } 0 \in A, \quad \delta_{0}=0 \text { if } 0 \notin A\right),
$$

and

$$
h(y)=\frac{e^{i y}-1}{i y} \text { if } y \neq 0, h(0)=1,
$$

we get

$$
\begin{aligned}
\frac{1}{T} \int_{0}^{T}|f(t)-C|^{2} d t & =\int_{R_{1}}^{L} \int_{R_{1}}\left\{\frac{1}{T} \int_{0}^{T} e^{i t(s-v)} d t\right\} d \phi_{1}(s) \overline{d \phi_{1}(v)} \\
& =\int_{R_{1}}^{L} \int_{R_{1}}^{L} h(T(s-v)) \overline{d \phi_{1}(v)} d \phi_{1}(s) .
\end{aligned}
$$

But $\lim _{y \rightarrow+\infty} h(y)=0$, so that, by dominated convergence, $|h(y)| \leq 2$ and $\phi$ bounded, we have

$$
\lim _{T \rightarrow \infty} \frac{1}{T} \int_{0}^{T}|f(t)-C|^{2} d t=\int_{R_{1}} \overline{\phi_{1}(\{s\})} d \phi_{1}(s)=\sum_{\phi_{1}(\{s\}) \neq 0}\left|\phi_{1}(\{s\})\right|^{2},
$$

which shows that Lemma 3 follows for $t \rightarrow+\infty$ from Lemma 2. As $t \rightarrow-\infty$ the same argument holds except that then

$$
h(y)=\frac{1-e^{-i y}}{i y} \text { for } y \neq 0 .
$$

We can now state our main affirmative result for tractable $\gamma$, (that is, $\gamma \sigma$ additive and bounded over $\mathcal{F}$, the finite algebra generated by the intervals of $R_{2}$.)

THEOREM l. If $\gamma$ as defined by $E(A)$ and $u_{0}$, and $P$ is tractable, then each of the following three statements implies every other one:

$$
\left\|P u_{t}\right\|^{2} \rightarrow C \text { densely as } t \rightarrow+\infty
$$


(b)

$$
\left\|P u_{t}\right\|^{2} \rightarrow C \text { densely as } t \rightarrow-\infty
$$

(c)

$$
\tilde{\gamma}(\{s\})=\gamma\left(D_{s}\right)=0 \text { for } s \neq 0 \text { and } \tilde{\gamma}(\{0\})=\gamma\left(D_{0}\right)=C \text {. }
$$

This theorem is an obvious consequence of Lemma 3 applied to equation (2.2). Because this answer for tractable $\gamma$ lies so close to the surface, it is desirable to see what can happen if $\gamma$ is not tractable. This appears of particular importance for quantum physics in studying transitions, for if $\gamma$ is tractable then $(a) \Longleftrightarrow(b)$ in our theorem shows in a certain sense that no change can take place in the probability of the system being in the situation of which $P$ is the projection. Unfortunately, as one might suspect, if $\gamma$ is not tractable there seems to be no general procedure applicable to all possible cases. We shall content ourselves by giving in the next section a few examples of what can happen.

3. Examples. The first example is constructed for the Hilbert space $X=$ $L_{2}(-\pi, \pi)$, using Fourier series, and here we get a nontractable $\gamma$, being neither $\sigma$-additive nor bounded over $\gamma$. We also have $\left\|P u_{t}\right\|^{2} \rightarrow 0$ densely as $t \rightarrow-\infty$ and $\left\|P u_{t}\right\|^{2} \longrightarrow 1=\left\|u_{0}\right\|^{2}$ densely as $t \rightarrow+\infty$ here, so that (a) no longer is equivalent to $(b)$ as in Theorem 1 . The second example gives a nontractable $\gamma$ for $X=L_{2}(-\infty, \infty)$, but here this $\gamma$ "blows up at $\infty$," whereas in the first example it is only in the neighborhood of the main diagonal $D_{0}$ that $\gamma$ behaves badly. The different behavior of $\gamma$ in these two examples illustrates the difficulty of getting any general result like Theorem 1 for nontractable $\gamma$.

EXAMPLE 1. Let

$$
X=L_{2}(-\pi, \pi), v_{p}(x)=\frac{e^{i p x}}{\sqrt{2 \pi}},
$$

so that $\left\{v_{p}\right\}$ is a complete orthonormal system for $X$, and let the projection $P$ be defined by

$$
P u=\sum_{p=1}^{\infty}\left(u, v_{p}\right) v_{p},
$$

convergent in $X$ norm. Also let

$$
[E(A) u](x)=\chi_{A}(x) u(x) \text { for } A \in B_{1},
$$


$\chi_{A}$ being the characteristic function of $A$, so $E(A)$ is a spectral measure. Finally let $u_{0}=v_{0}$. The $\gamma$ which $E(A), P$, and $u_{0}$ define is not tractable, being neither $\sigma$-additive nor bounded over $\mathcal{F}$; $\gamma$ is given for subintervals $A$ and $B$ of $[-\pi, \pi]$ by

$$
\begin{aligned}
\gamma(A \times B)= & \frac{1}{4 \pi} \mu_{1}(A \cap B) \\
& \quad-\frac{1}{2(2 \pi)^{2}}\left\{\mu_{2}(A \times B)+\int \frac{1+\cos (y-x)}{i \sin (y-x)} d \mu_{2}(x, y)\right\}
\end{aligned}
$$

where $\mu_{2}$ is ordinary Lebesgue measure on $R_{2}$ and the integral ranges over $A \times B-(A \cap B) \times(A \cap B)$;

$$
\begin{gathered}
\left\|P u_{t}\right\|^{2}=1 / 2 \\
+\frac{1}{2(2 \pi)^{2}} \int_{-\pi}^{\pi} \int_{-\pi}^{\pi}\left\{-\cos t(y-x)+\frac{[1+\cos (y-x)] \sin t(y-x)}{\sin (y-x)}\right\} d y d x
\end{gathered}
$$

and in the ordinary sense, which implies dense convergence,

$$
\left\|P u_{t}\right\|^{2} \rightarrow 0 \text { as } t \rightarrow-\infty \text { and } \rightarrow 1=\left\|u_{0}\right\|^{2} \text { as } t \rightarrow+\infty \text {. }
$$

To prove these assertions we shall first establish (3.1). Here

$$
\begin{aligned}
\gamma(A \times B) & \left.=\left(P E(A) u_{0}, E(B) u_{0}\right)=\sum_{p=1}^{\infty}\left(\int_{A} u_{0}(x) \overline{v_{p}(x)} d x\right) \overline{\left(\int_{B} u_{0}(y) \overline{v_{p}(y) d y}\right.}\right) \\
& =\lim _{n \rightarrow \infty} \frac{1}{(2 \pi)^{2}} \int_{A \times B}\left(\sum_{p=1}^{n} e^{i p(y-x)}\right) d \mu_{2}(x, y) \\
& =\lim _{n \rightarrow \infty} \frac{1}{(2 \pi)^{2}} \int_{A \times B} \frac{e^{i(y-x)}-e^{i(n+1)(y-x)}}{1-e^{i(y-x)}} d \mu_{2}(x, y) .
\end{aligned}
$$

Now if $A$ and $B$ are subintervals of $[-\pi, \pi]$ having $A \cap B=\phi$ (or actually containing at most one point), then

$$
\left(1-e^{i(y-x)}\right)^{-1} \in L_{1}\left(A \times B, \mu_{2}\right)
$$

is easily verified. Ilence rotating coordinates and applying the Riemann- 
Lebesgue lemma to evaluate the previous limit for $\gamma$, we get

$$
\begin{aligned}
\gamma(A \times B) & =\frac{1}{(2 \pi)^{2}} \int_{A \times B} \frac{e^{i(y-x)}}{1-e^{i(y-x)}} d \mu_{2}(x, y) \\
& =\frac{1}{(2 \pi)^{2}} \int_{A \times B} \frac{e^{i(y-x)}-1}{\left|1-e^{i(y-x)}\right|^{2}} d \mu_{2}(x, y) \\
& =\frac{1}{(2 \pi)^{2}} \int_{A \times B} \frac{\cos (y-x)-1+i \sin (y-x)}{2(1-\cos (y-x))} d \mu_{2}(x, y) .
\end{aligned}
$$

Thus for disjoint intervals $A$ and $B$ we have

$$
\gamma(A \times B)=-\frac{1}{2(2 \pi)^{2}} \int_{A \times B}\left\{1+\frac{1+\cos (y-x)}{i \sin (y-x)}\right\} d \mu_{2}(x, y) .
$$

At the other extreme, for interval $A=B$, using the fact that $\gamma(A \times A)$ is real, we can verify

$$
\begin{aligned}
\gamma(A \times A)= & \lim _{n \rightarrow \infty} \frac{1}{(2 \pi)^{2}} \int_{A \times A} \frac{e^{i(y-x)}-e^{i(n+1)(y-x)}}{1-e^{i(y-x)}} d \mu_{2}(x, y) \\
= & \lim _{n \rightarrow \infty}\left(-\frac{1}{2(2 \pi)^{2}}\right) \int_{A \times A}\{1-\cos n(y-x) \\
& \left.-[1+\cos (y-x)] \frac{\sin (n(y-x))}{\sin (y-x)}\right\} d \mu_{2}(x, y) .
\end{aligned}
$$

Rotating coordinates and using the Riemann-Lebesgue lemma and known properties of the Dirichlet kernel to evaluate this limit, we get

$$
\gamma(A \times A)=-\frac{1}{2(2 \pi)^{2}} \mu_{2}(A \times A)+\frac{1}{4 \pi} \mu_{1}(A) .
$$

Finally, (3.3), (3.4), and the finite additivity of $\gamma$ over $\mathcal{F}$ imply (3.1) as desired.

Using (3.1) in (2.1) and noting the obvious cancellations we readily see that (3.2) results, with the integrand now bounded, unlike (3.1). But from (3.2) 
by the same evaluation leading to (3.4), we get

$$
\left\|P u_{t}\right\|^{2} \rightarrow \frac{1}{2}+\frac{1}{2}=1=\left\|u_{0}\right\|^{2} \text { as } t \rightarrow+\infty
$$

and

$$
\left\|P u_{t}\right\|^{2} \rightarrow \frac{1}{2}-\frac{1}{2}=0 \text { as } t \rightarrow-\infty
$$

as desired.

It only remains to show from (3.1) that $\gamma$ is neither bounded nor $\sigma$-additive

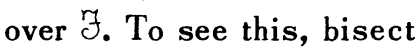

$$
[-\pi, \pi] \times[-\pi, \pi]=C_{0}
$$

into four equal closed squares, bisect those of these squares whose diagonals are segments of $D_{0}$, and continue the operation to get a sequence of squares $F_{j, p}$ above the main diagonal, explicitly

$$
F_{j, p}=\left[(2 \pi) \frac{2(j-1)}{2^{p}}-\pi,(2 \pi) \frac{2 j-1}{2^{p}}-\pi\right] \times\left[(2 \pi) \frac{2 j-1}{2^{p}}-\pi,(2 \pi) \frac{2 j}{2^{p}}-\pi\right]
$$

for $1 \leq j \leq 2^{p-1}$ and $1 \leq p$. The open subset

$$
V=\left\{(x, y) \in C_{0} \mid y>x\right\}
$$

of $C_{0}$ clearly has $[1+\cos (y-x)] / \sin (y-x)$ integrable Lebesgue over $V$, with

$$
\int_{V} \frac{1+\cos (y-x)}{\sin (y-x)} d \mu_{2}(x, y)=+\infty .
$$

Thus (3.1) and the $\sigma$-additivity of the Lebesgue integral, since

$$
V \subseteq \bigcup_{p=1}^{\infty} \bigcup_{j=1}^{2^{p-1}} F_{j, p},
$$

show that

$$
+\infty=\lim _{N \rightarrow \infty} d\left[\gamma\left(\bigcup_{p=1}^{N}{\underset{j}{2^{p-1}}}_{j} F_{j, p}\right)\right],
$$


so $\gamma$ is not bounded over $\mathcal{F}$.

It is only slightly more complicated to see that $\gamma$ is also not $\sigma$-additive over 7. For this, define the upper right corner square

$$
L_{p}=\left[\pi-\frac{2 \pi}{2^{p}}, \pi\right] \times\left[\pi-\frac{2 \pi}{2^{p}}, \pi\right]
$$

of $C_{0}$ and also

$$
K_{p}=[-\pi, \pi] \times\left[-\pi, \pi-\frac{2 \pi}{2^{p}}\right]
$$

Define $f(p)$ as the least integer $k \geq p+1$ having

$$
d\left[\gamma\left({\underset{F}{j, q \subseteq L_{p}}}_{q \leq k}^{\cup} F_{j, q}\right)\right] \geq(10)^{p},
$$

such $k$ existing from (3.1) since

$$
\int_{V \cap L_{p}} \frac{1+\cos (y-x)}{\sin (y-x)} d \mu_{2}(x, y)=+\infty \text {. }
$$

Now define the sequence of sets $\mathbb{W}_{n} \in \mathcal{F}$ by taking $\mathbb{W}_{0}=\{(\pi, \pi)\}$, a point interval, and

$$
W_{2 p-1}=\bigcup_{q=1}^{f(p)^{2}} \bigcup_{j=1}^{q-1} F_{j, q}, \quad W_{2 p}=K_{p}, \text { for } p \geq 1
$$

These in turn define the disjoint sequence $\mathbb{W}_{n}^{\prime} \in \mathcal{F}$ by

$$
W_{n}^{\prime}=W_{n}-W_{n} n\left(\begin{array}{cc}
\bigcup_{m=0}^{\prime} & W_{m}^{\prime}
\end{array}\right)
$$

and we clearly see that

$$
\bigcup_{n=0}^{\infty} \mathbb{W}_{n}^{\prime}=\bigcup_{n=0}^{\infty} W_{n}=C_{0}=[-\pi, \pi] \times[-\pi, \pi]
$$

Also clearly $f(p) \leq f(p+1)$ and $K_{p} \subseteq K_{p+1}$, so we see that 


$$
\begin{aligned}
& \bigcup_{n=0}^{2 p} \mathbb{W}_{n}^{\prime}=\bigcup_{n=0}^{2 p} \mathbb{W}_{n}=W_{0} \cup K_{p} \cup\left(\bigcup_{q=1}^{f(p)}{ }^{2} \bigcup_{j=1}^{q-1} F_{j, q}\right) \\
& =W_{0} \cup\left(C_{0}-L_{p}\right) \cup\left(\bigcup_{F_{j, q} \subseteq L_{p}}^{\cup} F_{j, q}\right) \text {, } \\
& q \leq f(p)
\end{aligned}
$$

and similarly

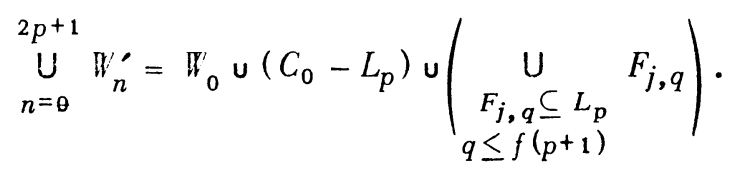

But hence $\gamma\left(W_{0}\right)=0$; and $d\left[\gamma\left(C_{0}-L_{p}\right)\right]=0$ from (3.1) makes

$$
\begin{aligned}
& d\left[\sum_{n=0}^{2 p} \gamma\left(W_{n}^{\prime}\right)\right]=d\left[\gamma\left(\underset{F_{j, q} \subseteq L_{p}}{U} F_{j, q}\right)\right] \geq(10)^{p} \\
& q \leq f(p)
\end{aligned}
$$

and likewise

$$
d\left[\sum_{n=0}^{2 p^{+1}} \gamma\left(W_{n}^{\prime}\right)\right]=d\left[\gamma\left(\underset{\substack{F_{j, q} \subseteq L_{p} \\ q \leq f(p+1)}}{U} F_{j, q}\right)\right] \geq d\left[\gamma\left(\underset{\substack{F_{j, q} \subseteq L_{p+1} \\ q \leq f(p+1)}}{U} F_{j, q}\right)\right] \geq(10)^{p+1}
$$

Thus we see that

$$
\lim _{N \rightarrow \infty} d\left[\sum_{n=0}^{N} \gamma\left(\mathbb{W}_{n}^{\prime}\right)\right]=+\infty
$$

although disjointly $\cup_{n=0}^{\infty} \mathbb{W}_{n}^{\prime}=C_{0} \in \mathcal{F}$ and $d\left[\gamma\left(C_{0}\right)\right]=0$ by (3.1), so $\gamma$ is not $\sigma$-additive over $\mathcal{F}$.

This completes our discussion of Example 1.

ExamPLe 2. Let

$$
X=L_{2}\left(R_{1}\right), \quad[E(A) u](x)=\chi_{A}(x) u(x)
$$


for $u \in X$ and $A \in B_{1}$ as in Example 1 ,

$$
\hat{u}(\omega)=\underset{L \rightarrow \infty}{\operatorname{norm} \operatorname{limit}} \frac{1}{\sqrt{2 \pi}} \int_{-L}^{L} e^{i \omega x} u(x) d x,
$$

the Fourier transform of $u$, and $\hat{u} \leftrightarrow u$ is a Hilbert space isomorphism of X onto X. Let

$$
[\widehat{P} \bar{u}](\omega)=\chi_{[-1,1]}(\omega) \hat{u}(\omega)
$$

define the orthogonal projection $P$ in $X$, and consider $u_{0} \in X$. Then $\left\|P u_{t}\right\|^{2} \rightarrow 0$ densely as $|t| \longrightarrow+\infty$ for every initial $u_{0} \in X$; $\gamma$ defined by $E(A), P$, and $u_{0}$ is tractable for $u_{0} \in L_{1}\left(R_{1}\right) \cap L_{2}\left(R_{1}\right)$;

$$
y(C)=\frac{1}{\pi} \int_{C}^{L} \frac{\sin (x-y)}{x-y} u_{0}(x) \overline{u_{0}(y)} d \mu_{2}(x, y)
$$

for all $C \in \mathcal{F}$ for $u_{0} \in L_{2}\left(R_{1}\right) \cap L_{1}\left(R_{1}\right)$, or for all bounded $C \in \mathcal{F}$ for any $u_{0} \in X$; but there exist $u_{0} \in X=L_{2}\left(R_{1}\right)$ for which $\gamma$ is not tractable, being unbounded over f.

We first need to verify (3.5). Here we note that for $R_{1}$ intervals $A$ and $B$,

$$
\begin{aligned}
\gamma(A \times B) & =\left(\operatorname{PE}(A) u_{0}, E(B) u_{0}\right)=\int_{-1}^{1}\left[\widehat{\chi_{A}} \widehat{u_{0}}\right](\omega) \overline{\left[\widehat{\chi_{B} u_{0}}\right](\omega) d \omega} \\
& =\frac{1}{2 \pi} \int_{-1}^{1} \int_{A} \int_{B} e^{i \omega(x-y)} u_{0}(x) \overline{u_{0}(y)} d x d y d \omega
\end{aligned}
$$

for any $u_{0} \in L_{2}\left(R_{1}\right) \cap L_{1}(A \cup B)$. The Fubini theorem now applies, yielding

$$
\begin{aligned}
y(A \times B) & =\frac{1}{2 \pi} \int_{A} \int_{B} \frac{e^{i(x-y)}-e^{-i(x-y)}}{i(x-y)} u_{0}(x) \overline{u_{0}(y)} d x d y \\
& =\frac{1}{\pi} \int_{A} \int_{B} \frac{\sin (x-y)}{x-y} u_{0}(x) \overline{u_{0}(y)} d x d y .
\end{aligned}
$$

Thus (3.5) follows as stated, either for $u_{0} \in L_{2}\left(R_{1}\right) \cap L_{1}\left(R_{1}\right)$ and any $C \in \mathcal{F}$, or for any $u_{0} \in L_{2}\left(R_{1}\right)$ and bounded $C \in \mathcal{F}$.

Now from (3.5) for $u_{0} \in L_{2}\left(R_{1}\right) \cap L_{1}\left(R_{1}\right)$, it is clear that $\gamma$ is tractable and 
$\left\|P u_{t}\right\|^{2} \rightarrow 0$ densely as $|t| \rightarrow \infty$, since

$$
\tilde{\gamma}(\{s\})=\gamma\left(D_{s}\right)=0
$$

for all real $s$. For general initial $u_{0} \in L_{2}\left(R_{1}\right)$, consider $u_{0}^{\prime} \in L_{2}\left(R_{1}\right) \cap L_{1}\left(R_{1}\right)$ and let $u_{t}=U_{t} u_{0}$ and $u_{t}^{\prime}=U_{t} u_{0}^{\prime}$, so that we have

$$
\left\|P u_{t}-P u_{t}^{\prime}\right\| \leq\left\|u_{t}-u_{t}^{\prime}\right\|=\left\|u_{0}-u_{0}^{\prime}\right\|
$$

Thus

$$
\frac{1}{2 T} \int_{-T}^{T}\left\|P u_{t}\right\| d t \leq \frac{1}{2 T} \int_{-T}^{T}\left\|P u_{t}^{\prime}\right\| d t+\left\|u_{0}-u_{0}^{\prime}\right\|
$$

But $\left\|P u_{t}^{\prime}\right\|^{2} \rightarrow 0$ densely as $|t| \rightarrow+\infty$ implies the same for $\left\|P u_{t}^{\prime}\right\|^{1 / 2}$, so

$$
\lim _{T \rightarrow \infty} \frac{1}{2 T} \int_{-T}^{T}\left\|P u_{t}^{\prime}\right\| d t=0
$$

by Lemma 2 , and hence

$$
\limsup _{T \rightarrow \infty}\left(\frac{1}{2 T} \int_{-T}^{T}\left\|P u_{t}\right\| d t\right) \leq\left\|u_{0}-u_{0}^{\prime}\right\|
$$

Thus the density of $L_{2}\left(R_{1}\right) \cap L_{1}\left(R_{1}\right)$ in $L_{2}\left(R_{1}\right)$ shows

$$
\lim _{T \rightarrow \infty} \frac{1}{2 T} \int_{-T}^{T}\left\|P u_{t}\right\| d t=0
$$

so Lemma 2 shows that $\left\|P u_{t}\right\|^{1 / 2}$ and hence $\left\|P u_{t}\right\|^{2} \rightarrow 0$ densely as $|t| \rightarrow+\infty$ for every initial $u_{0} \in L_{2}\left(R_{1}\right)$.

It only remains to show the existence of $u_{0} \in L_{2}\left(R_{1}\right)$ for which $\gamma$ is not tractable. First notice that $(3.5)$ for bounded $C \in \mathcal{F}$ implies, for the total variation of $\gamma$ over F,

$$
\operatorname{var} \gamma=\frac{1}{\pi} \int_{R_{2}}\left|\frac{\sin (x-y)}{x-y}\right|\left|u_{0}(x)\right|\left|u_{0}(y)\right| d \mu_{2}(x, y)
$$

for $u_{0} \in L_{2}\left(R_{1}\right)$, so that if $\gamma$ is always tractable this is always finite for every $u_{0}=u \in L_{2}\left(R_{1}\right)$. Hence 


$$
\begin{aligned}
& \int_{R_{2}}\left|\frac{\sin (x-y)}{x-y}\right||u(x)||v(y)| d \mu_{2}(x, y) \\
& \quad \leq \int_{R_{2}}\left|\frac{\sin (x-y)}{x-y}\right|(|u(x)|+|v(x)|)(|u(y)|+|v(y)|) d \mu_{2}(x, y)
\end{aligned}
$$

is also finite for every $u$ and $v \in L_{2}\left(R_{1}\right)$. Thus

$$
[F(u)](y)=\frac{1}{\pi} \int_{R_{1}}\left|\frac{\sin (y-x)}{y-x}\right| u(x) d x,
$$

clearly existent, has $[F(u)](y) v(y) \in L_{1}\left(R_{1}\right)$ by the Fubini theorem for every $u$ and $v \in L_{2}\left(R_{1}\right)$, so $F(u) \in L_{2}\left(R_{1}\right)$ also. Likewise $\left(F u_{s} v\right)=(u, F v)$ follows from the Fubini theorem, so $F$ is a symmetric, everywhere defined, linear operator on $L_{2}\left(R_{1}\right)$, and hence a bounded Hermitian operator. Now define $f \in L_{2}\left(R_{1}\right)$ uniquely by requiring

$$
\hat{f}(\omega)=\frac{2}{\pi}\left|\frac{\sin \omega}{\omega}\right| \quad \text { in } L_{2}\left(R_{1}\right),
$$

and note that

$$
[F(u)](y)=\frac{1}{\sqrt{2 \pi}} \int_{R_{1}} \hat{f}(y-x) u(x) d x .
$$

Since $F$ is bounded from $L_{2}\left(R_{1}\right)$ into $L_{2}\left(R_{1}\right)$, we can by using the density of $L_{2}\left(R_{1}\right) \cap L_{1}\left(R_{1}\right)$ in $L_{2}\left(R_{1}\right)$ for approximation, get the usual convolution result

$$
[\widehat{F(u)}](\omega)=f(\omega) \hat{u}(\omega) \text {. }
$$

Thus

$$
\|F(u)\|=\|\widehat{F(u)}\|
$$

and $F$ bounded makes

$$
\underset{\omega \in R_{1}}{\text { ess sup }}|f(\omega)|=\|F\|<+\infty,
$$

so $f \in L_{\infty}\left(R_{1}\right) \cap L_{2}\left(R_{1}\right)$. But thus the easy extension of Bochner's theorem $\left[1, \mathrm{p} .20\right.$, Theorem 9] from $L_{1}\left(R_{1}\right)$ to $L_{p}\left(R_{1}\right)$, and $p=2$ in our case, requires our nonnegative $\hat{f}(\omega)$ to have 


$$
\sqrt{\frac{2}{\pi}}\left|\frac{\sin \omega}{\omega}\right|=\hat{f}(\omega) \in L_{1}\left(R_{1}\right),
$$

contradicting

$$
\left|\frac{\sin \omega}{\omega}\right| \notin L_{1}\left(R_{1}\right) \text {. }
$$

Thus in (3.6) we must have var $\gamma=+\infty$ and $\gamma$ nontractable for some $u_{0} \in L_{2}\left(R_{1}\right)$. This completes our discussion of Example 2.

\section{REFERENCEE}

1. S. Bochner and K. Chandrasekharan, Fourier integrals, Ann. of Math. Studies, No. 19, Princeton, 1949.

2. H. Cramé, Random variables and probability distributions, Cambridge Tract No. 36, Cambridge University Press, Cambridge, England, 1937.

3. P. R. Halmos, Measure theory, D. Van Nostrand Co., New York, 1950.

4. - Introduction to Hilbert space, Chelsea Publishing Co., New York, 1951. 



\section{Pacific Journal of Mathematics}

\section{Vol. 5, No. $1 \quad$ September, 1955}

Frank Herbert Brownell, III, Flows and noncommuting projections on

Hilbert space ................................... 1

H. E. Chrestenson, A class of generalized Walsh functions ............. 17

Jean Bronfenbrenner Crockett and Herman Chernoff, Gradient methods of maximization ................................... 33

Nathan Jacob Fine, On groups of orthonormal functions. I .......... 51

Nathan Jacob Fine, On groups of orthonormal functions. II ............ 61

Frederick William Gehring, A note on a paper by L. C. Young .......... 67

Joachim Lambek and Leo Moser, On the distribution of Pythagorean

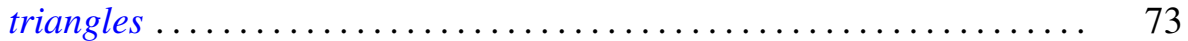

Roy Edwin Wild, On the number of primitive Pythagorean triangles with

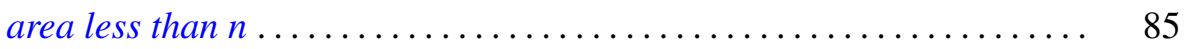

R. Sherman Lehman, Approximation of improper integrals by sums over multiples of irrational numbers ........................ 93

Emma Lehmer, On the number of solutions of $u^{k}+D \equiv w^{2}(\bmod p) \ldots 103$

Robert Delmer Stalley, A modified Schnirelmann density............... 119

Richard Allan Moore, The behavior of solutions of a linear differential equation of second order............................. 125

William M. Whyburn, A nonlinear boundary value problem for second order differential systems. 